\title{
The effect of propofol on emergence agitation in children receiving sevoflurane for adenotonsillectomy
}

\author{
Cheol Jin Lee ${ }^{1}$, Sang Eun Lee, ${ }^{1, *}$, Min Kyung $\mathrm{Oh}^{2}$, Chee Mahn Shin ${ }^{1}$, Young Jae Kim¹, \\ Young Kyun Choe ${ }^{1, *}$, Soon Ho Cheong ${ }^{1}$, Kun Moo Lee ${ }^{1}$, Jeong Han Lee ${ }^{1}$, Se Hun Lim ${ }^{1}$, \\ Young Hwan $\mathrm{Kim}^{1}$, and Kwang Rae Cho ${ }^{1}$ \\ Departments of ${ }^{1}$ Anesthesiology and Pain Medicine, ${ }^{2}$ Clinical Trial Center, Busan Paik Hospital, College of Medicine, Inje University, \\ Busan, Korea
}

Background: The administration of a single dose of propofol is reported to be effective in decreasing the incidence and severity of emergence agitation (EA) in children following sevoflurane anesthesia. The aim of this study was to investigate the clinical usefulness of a single dose of propofol $1 \mathrm{mg} / \mathrm{kg}$ at the end of adenotonsillectomy for reducing the incidence of EA after sevoflurane anesthesia.

Methods: Ninety children, aged 3-8 years, undergoing adenotonsillectomy were randomized into two groups: the propofol group $(n=45)$ and the saline group $(n=45)$, of which 88 children completed the study. Anesthesia was maintained with sevoflurane $2-2.5$ vol\% and nitrous oxide/oxygen (50\%/50\%). At the completion of adenotonsillectomy, the propofol group patients were given $1 \mathrm{mg} / \mathrm{kg}$ of propofol and the saline group patients were given saline $0.1 \mathrm{ml} / \mathrm{kg}$ in the same volume. The incidence of EA was assessed with Aono's four point scale and the severity of EA was assessed with pediatric anesthesia emergence delirium (PAED) scale at 5 min (T5), 15 min (T15) and $30 \mathrm{~min}$ (T30) after emergence.

Results: Of the 88 patients, the incidence of EA at T5, T15 and T30 was $61.4 \%, 27.3 \%$, and $4.5 \%$ in the propofol group while in the saline group was $68.2 \%, 29.5 \%$, and $9.1 \%$, respectively. The incidence and severity of EA were not found to be significantly different between the two groups, but the scales in each group decreased significantly over time.

Conclusions: The administration of propofol $1 \mathrm{mg} / \mathrm{kg}$ at the end of surgery did not have any significant effect in reducing the incidence and severity of EA in children undergoing adenotonsillectomy under sevoflurane anesthesia. (Korean J Anesthesiol 2010; 59: 75-81)

Key Words: Child, Emergence agitation, Propofol, Sevoflurane.

Received: January 21, 2010. Revised: 1st, February 12, 2010; 2nd, March 21, 2010. Accepted: May 17, 2010.

Corresponding author: *[Present address] Sang Eun Lee, M.D., Department of Anesthesiology and Pain Medicine, Haeundae Paik Hospital, College of Medicine, Inje University, 1435, Ja-dong, Haeundae-gu, Busan 612-030, Korea. Tel: 82-51-797-0425, Fax: 82-51-797-0499, E-mail: Painlee@medimail.co.kr *[Present address] Young Kyun Choe, M.D., Department of Anesthesiology and Pain Medicine, Haeundae Paik Hospital, College of Medicine, Inje University, 1435, Ja-dong, Haeundae-gu, Busan 612-030, Korea.

This is a theisis for a master's degree.

(c) This is an open-access article distributed under the terms of the Creative Commons Attribution Non-Commercial License (http:// creativecommons.org/licenses/by-nc/3.0/), which permits unrestricted non-commercial use, distribution, and reproduction in any medium, provided the original work is properly cited. 


\section{Introduction}

Sevoflurane is widely used as an anesthetic agent for children because of its less pungent nature and also as it has a lower solubility and greater hemodynamic stability than the other potent inhaled anesthetics [1]. However, sevoflurane may have a greater incidence of emergence agitation (EA) in preschool aged children [2].

EA is characterized by non-purposeful restlessness and agitation, thrashing, crying or moaning, disorientation, and incoherence. Although generally self-limiting, EA can be dangerous and may occasionally result in physical harm to children. Sometimes sedative or analgesic drugs are used in postanesthesia care units to treat EA. Therefore, recovery time can be prolonged and adverse events may result in these children [3].

Propofol is a short-acting sedative and hypnotic agent. Generally, propofol is used in children for its sedative action as well as for induction and maintenance of general anesthesia [4]. A recent study showed that continuous intravenous infusion of propofol decreased the incidence of EA in the children undergoing tonsillectomy [5] and the administration of $1 \mathrm{mg} / \mathrm{kg}$ propofol at the end of procedure decreased the incidence of EA in children undergoing magnetic resonance imaging (MRI) [6].

The administration of a single dose of propofol $(1 \mathrm{mg} / \mathrm{kg})$ at the end of surgery was also effective in decreasing the incidence of EA in children undergoing strabismus surgery under sevoflurane [7]. However, adenotonsillectomy is more distressing to children than strabismus surgery. The effect of administration of $1 \mathrm{mg} /$ $\mathrm{kg}$ propofol in children following adenotonsillectomy may work differently from those undergoing MRI as reported by previous studies $[6,7]$. Therefore, the aim of this study was to investigate the clinical usefulness of a single dose of propofol at the end of adenotonsillectomy in reducing the incidence and severity of EA after sevoflurane anesthesia.

\section{Materials and Methods}

Children, between the ages of 3 and 8 years, with American Society of Anesthesiologists physical status I, who were scheduled to undergo adenotonsillectomy without myringotomy at the department of otolaryngology, were prospectively enrolled in this study. Prior to their enrollment, institutional review board approval and informed written parental consent were obtained. Children with mental, neurologic disease, or those using sedative medication were excluded.

Ninety children joined the study at our hospital between June 2007 and April 2009. A computer generated randomization was placed in sealed envelopes, which were opened on the day of the scheduled operation. Forty five children were randomly assigned to the propofol group and another forty five to the saline group. One child from the propofol group was excluded from the study because of intra-operative ST depression on EKG and one other child from the saline group was also excluded due to bleeding at the surgical region after extubation. Thus, the data for this study were collected from eighty-eight patients. Demographic profile, duration of surgery, duration of sevoflurane administration, and recovery characteristics are shown in Table 1, which shows no significant difference between the two groups.

Children were in fasting state for 8 hours and received $1 \mathrm{mg} / \mathrm{kg}$ of intravenous thiopental sodium before entering the operating room. An electrocardiogram, pulse oximeter and noninvasive arterial blood pressure monitor were attached. Anesthesia was induced with $5 \mathrm{mg} / \mathrm{kg}$ of intravenous thiopental sodium and 0.5 $\mathrm{mg} / \mathrm{kg}$ of intravenous atracurium. Orotracheal intubation was performed. Anesthesia was maintained with $\mathrm{O}_{2} 2 \mathrm{~L} / \mathrm{min}, \mathrm{N}_{2} \mathrm{O}$ $2 \mathrm{~L} / \mathrm{min}$ and sevoflurane $2-2.5$ vol\%. Mechanical ventilation was performed to maintain an end-tidal $\mathrm{CO}_{2}$ between 30 and 35 mmHg. About 20 minutes prior to the end of surgery, ketorolac $1 \mathrm{mg} / \mathrm{kg}$ was administered intravenously to each patient for postoperative pain control.

At the completion of the operation, following the discontinuation of sevoflurane and nitrous oxide, children in the propofol group were given $1 \mathrm{mg} / \mathrm{kg}$ of propofol and children in the saline group were given the same volume of normal saline, $0.1 \mathrm{ml} / \mathrm{kg}$. The orotracheal tube was removed when patients demonstrated spontaneous regular respiratory pattern and the rate of train of four (TOF) was higher than 0.8 by a nerve stimulator (TOF-Watch ${ }^{\circledR}$, Organon, Ireland). Anticholinesterase agents that reverse the effect of neuromuscular blockade were not administered. Children were transferred to the post-anesthesia care unit (PACU) and one of their parents accompanied them at the PACU until discharge.

An anesthesiologist, who was blinded to the treatment

Table 1. Demographic and Recovery Characteristics

\begin{tabular}{lccc}
\hline & $\begin{array}{c}\text { Propofol group } \\
(\mathrm{n}=44)\end{array}$ & $\begin{array}{c}\text { Saline group } \\
(\mathrm{n}=44)\end{array}$ & P value \\
& $26 / 18$ & $25 / 19$ & 0.831 \\
Sex (M/F) & $5.8 \pm 1.9$ & $6.3 \pm 1.6$ & 0.204 \\
Age (yr) & $22.4 \pm 7.2$ & $24.2 \pm 6.4$ & 0.192 \\
Weight (kg) & $37.7 \pm 12.4$ & $34.5 \pm 9.0$ & 0.167 \\
Duration of surgery (min) & $43.6 \pm 13.2$ & $42.5 \pm 10.8$ & 0.585 \\
Duration of sevoflurane & & & \\
administration (min) & $13.7 \pm 3.8$ & $12.2 \pm 4.1$ & 0.188 \\
Duration of extubation (min) & $24.2 \pm 5.0$ & $25.0 \pm 6.1$ & 0.516 \\
Duration of PACU stay (min) & $24.05 \%$ & $6(13.6 \%)$ & 0.737 \\
PONV (\%) & $4(9.1 \%)$ & 6
\end{tabular}

Values are presented as mean \pm SD or number. Children in the propofol group were given $1 \mathrm{mg} / \mathrm{kg}$ of propofol and children in the saline group were given the same volume of normal saline, $0.1 \mathrm{ml} /$ kg. PACU: post-anesthesia care unit, PONV: postoperative nausea and vomiting. 
allocation, evaluated the incidence of EA using Aono's four point scale (Appendix 1) and severity of EA using pediatric anesthesia emergence delirium (PAED, Appendix 2) scale, at 5 (T5), 15 (T15) and 30 (T30) minutes (min) after emergence. Emergence time was defined as the time of first response to command or eye opening on command after extubation. Aono's four point scale of 1 and 2 were considered as the absence of EA, and scales of 3 and 4 were considered as the presence of EA. PAED scale was used to assess the severity of EA. Pain was evaluated using children and infants postoperative pain (CHIPP, Appendix 3) scale and consciousness was evaluated using Ramsay sedation scale (Appendix 4) in the PACU by the same anesthesiologist at 5,15 and 30 minutes after emergence. The incidence of postoperative nausea and vomiting (PONV) was also noted.

The following time intervals were recorded: the duration of surgery (from the time of opening mouth to the completion of the procedure), duration of sevoflurane administration (from the start of induction until discontinuation of sevoflurane), duration of extubation (from the time of discontinuation of sevoflurane to the removal of endotracheal tube) and duration of PACU

Table 2. Incidence of Emergence Agitation

\begin{tabular}{cccc}
\hline & $\begin{array}{c}\text { Propofol group } \\
(\mathrm{n}=44)\end{array}$ & $\begin{array}{c}\text { Saline group } \\
(\mathrm{n}=44)\end{array}$ & P value \\
\hline T5 & $27(61.4 \%)$ & $30(68.2 \%)$ & 0.655 \\
T15 & $12(27.3 \%)$ & $13(29.5 \%)$ & 0.815 \\
T30 & $2(4.5 \%)$ & $4(9.1 \%)$ & 0.672 \\
\hline
\end{tabular}

Values are presented as number (\%). T5, T15, and T30 represent the time after emergence (min). Children in the propofol group were given $1 \mathrm{mg} / \mathrm{kg}$ of propofol and children in the saline group were given the same volume of normal saline, $0.1 \mathrm{ml} / \mathrm{kg}$.

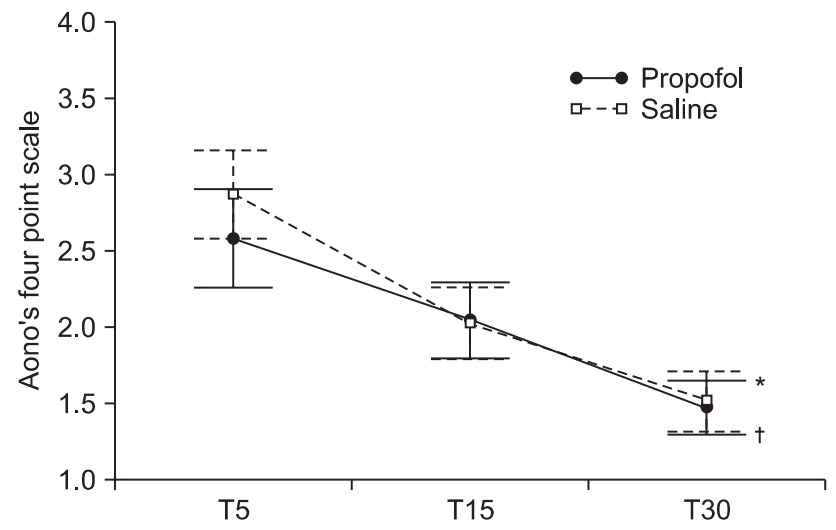

Fig. 1. Aono's four point scale at 5 (T5), 15 (T15) and 30 (T30) minutes after emergence. Aono's four point scales significantly decreased over time $(\mathrm{P}<0.001)$ and there was a statistically significant time effect in each group $\left({ }^{*} \mathrm{P}<0.001\right.$ in the propofol group and ${ }^{\dagger} \mathrm{P}<0.001$ in the saline group). Group effect averaged across time did not show significant difference $(\mathrm{P}=0.465)$ and the interaction effect of group and time was also not statistically significant $(P=0.196)$. Values are presented as mean \pm SEM. stay (from arrival to the PACU until discharge). Children were discharged from the PACU to a ward when the modified Aldrete score was more than 9 without agitation and vomiting.

Statistical analysis was performed using MedCalc for Windows version 11.0 (MedCalc Software, Mariakerke, Belgium). A sample size of 40 in each group was calculated to achieve $80 \%$ power to detect a decrease in the incidence of agitation from $60 \%$ to $30 \%$ with a significance P value 0.05 . Ninety patients were included. Continuous variables such as age, weight, duration of surgery, duration of sevoflurane administration, duration of extubation and duration of PACU stay were reported as mean \pm SD and analyzed using independent samples t-test. Categorical variables were compared using chi-square test for gender and the incidence of EA and using Fisher's exact test for the incidence of PONV. Serial measurements such as Aono's four point scale, PAED scale, CHIPP scale, and Ramsay sedation scale were analyzed by repeated measures analysis of variance. A P value less than 0.05 was considered statistically significant.

\section{Results}

The mean scores of the Aono's four point scale at T5, T15 and T30 were $2.6 \pm 1.1,2.0 \pm 0.8$, and $1.5 \pm 0.6$ respectively in the propofol group while $2.9 \pm 1.0,2.0 \pm 0.8$, and $1.5 \pm 0.7$ in the saline group. The mean scores of the PAED scale at T5, T15 and T30 were $12.6 \pm 4.6,8.2 \pm 3.8$, and $5.0 \pm 3.1$ respectively in the propofol group while $13.8 \pm 4.7,8.0 \pm 3.9$, and $4.5 \pm 3.1$ in the saline group. The incidence and severity of EA at T5, T15, and T30 shows no significant difference between the two groups (Table 2, Fig. 1 and 2). CHIPP scale and Ramsay sedation scale

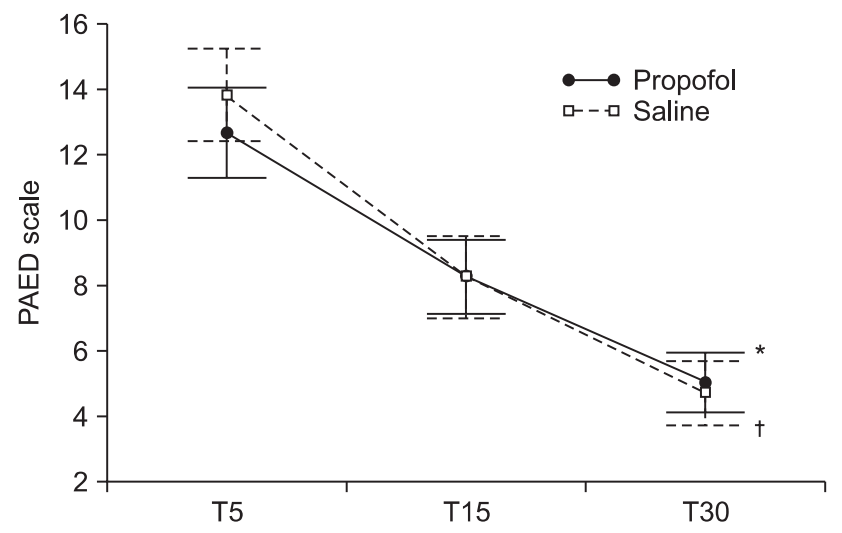

Fig. 2. Pediatric anesthesia emergence delirium (PAED) scale at 5 (T5), 15 (T15), and 30 (T30) minutes after emergence. PAED scales significantly decreased over time $(P<0.001)$ and there was a statistically significant time effect in each group $\left({ }^{*} \mathrm{P}<0.001\right.$ in the propofol group, ${ }^{\dagger} \mathrm{P}<0.001$ in the saline group). Group effect averaged across time did not show significant difference $(\mathrm{P}=0.709)$ and the interaction effect of group and time was not statistically significant $(P=0.093)$. Values are presented as mean \pm SEM. 


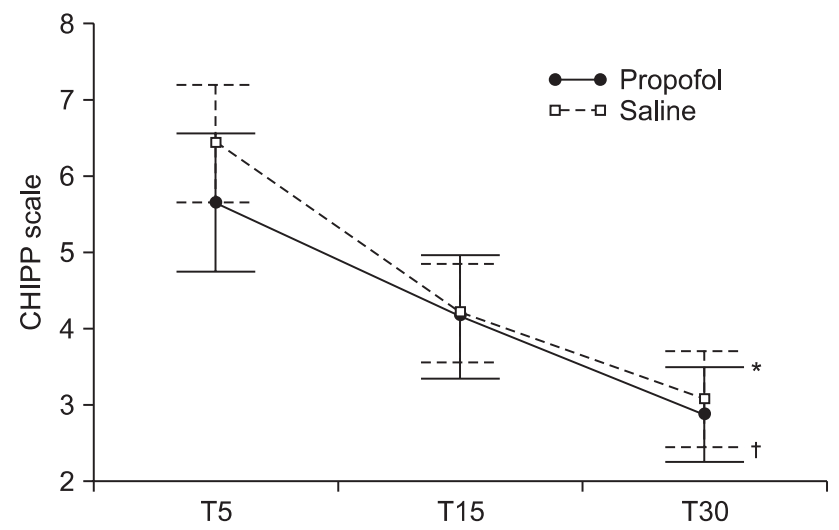

Fig. 3. Children and infants postoperative pain (CHIPP) scale at 5 (T5), 15 (T15), and 30 (T30) minutes after emergence. CHIPP scales significantly decreased by time $(\mathrm{P}<0.001)$ and there was a statistically significant time effect in each group $\left({ }^{*} \mathrm{P}<0.001\right.$ in propofol group, ${ }^{\dagger} \mathrm{P}<0.001$ in saline group). Group effect averaged across time did not show significant difference $(\mathrm{P}=0.428)$ and the interaction effect of group and time was not statistically significant $(\mathrm{P}$ $=0.312$ ). Values are presented as mean \pm SEM.

were similar in the two groups at T5, T15, and T30 (Fig. 3 and 4). Therefore, there were no difference of the intensity of pain and the level of consciousness between the two groups at 5,15 , and 30 min after emergence. Aono's four point scale, PAED scale and CHIPP scale in each group decreased significantly over time. All agitation episodes were self-limited.

$9.1 \%$ of the propofol group and $13.6 \%$ of the saline group had episodes of PONV, but there was no significant statistical difference between the two groups. No adverse events such as laryngospasm and oxygen desaturation episodes were recorded in any of the children. Time to discharge from the PACU was not significantly different between the two groups.

\section{Discussion}

Several previous studies have reported that sevoflurane anesthesia caused children to have a higher incidence of EA. Children anaesthetized with sevoflurane had a $67 \%$ incidence of postoperative agitation while halothane group had a $29.2 \%$ incidence [8]. Although the possible etiological factors of EA are post-anesthesia, surgery, rapid emergence, postoperative pain, age, preoperative anxiety, child temperament, and adjuvant medication, the causes for higher incidence of EA after sevoflurane are not fully understood. Because inhalational anesthetics with low blood solubility such as sevoflurane generally tend to cause a higher incidence of EA, rapid awakening in an unfamiliar environment has also been posited as a cause for this phenomenon.

Propofol allows for rapid emergence from general anesthesia and is not associated with a high incidence of EA in young

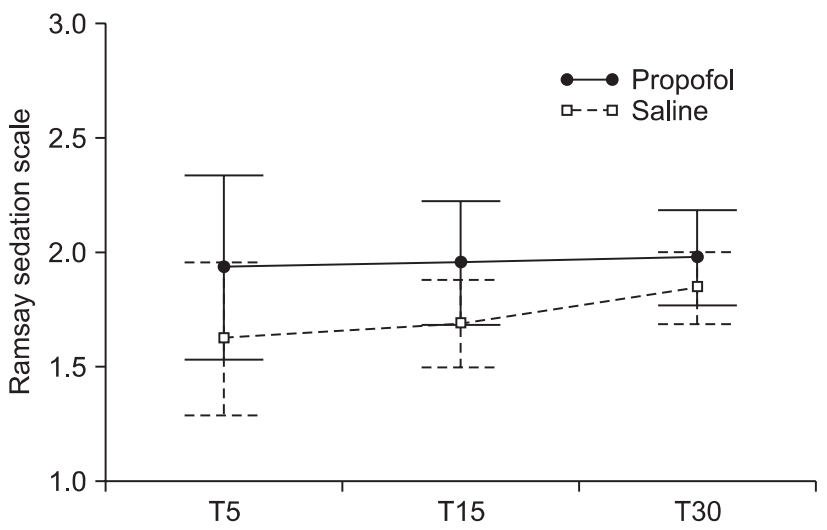

Fig. 4. Ramsay sedation scale at 5 (T5), 15 (T15), and 30 (T30) minutes after emergence. Ramsay sedation scales did not show significant difference over time $(P=0.436)$. Group effect and interaction effect of group and time were not statistically significant $(\mathrm{P}=0.116$ and 0.679$)$. Values are presented as mean $\pm \mathrm{SEM}$.

children [9]. Recovery from propofol anesthesia is smooth and delayed, so propofol anesthesia is associated with a lower incidence of EA compared to sevoflurane [5]. The decreased incidence of EA could be accounted for the residual sedative effect and euphoric effect of propofol in the early recovery period [10]. Based on previous studies, propofol seems to be effective in preventing EA and is dependent on the timing of administration [11]. Despite this fact, sevoflurane is considered the agent of choice for induction and maintenance of anesthesia for children and is widely used among pediatric anesthesiologists. A single dose of propofol at the end of surgery could delay or modify emergence and decrease the incidence of EA after sevoflurane anesthesia.

Abu-Shahwan's study [6] showed that the administration of sub-hypnotic doses of propofol at the end of sevoflurane general anesthesia was effective in decreasing the incidence and severity of EA in children undergoing MRI. Aouad et al. [7] reported that the administration of a single dose of propofol $1 \mathrm{mg} / \mathrm{kg}$ after discontinuation of sevoflurane at the end of surgery in children undergoing strabismus surgery significantly decreased the incidence of EA and improved patient satisfaction, and they concluded that the delayed emergence from anesthesia reduced PAED scales without delaying discharge from the PACU. However, Abu-Shahwan's case did not include painful procedures and the characteristics of strabismus surgery in Aouad's research were different from those of adenotonsillectomy in our study.

We supposed that the effect of propofol on EA in patients undergoing adenotonsillectomy may be different from that in strabismus patients, and then tried to investigate the effect of 1 $\mathrm{mg} / \mathrm{kg}$ propofol administration on the incidence and severity of EA at the end of adenotonsillectomy after discontinuation of sevoflurane. The single dose of propofol administration didn't 
have significant effect on the decrease of the incidence and severity of EA in the present study.

The percentage of EA at T5 was $61.4 \%$ in the propofol group and $68.2 \%$ in the saline group using Aono's four point scale in our study. The definition of EA in the study included children who were inconsolably crying as well as restless and therefore the incidence of EA might increase from 10 to $30 \%$ compared to other studies [12]. The PAED scale is a reliable and valid measurement that may minimize measurement errors in the clinical evaluation of EA [13]. However, the calculation of the incidence of EA with this scale is difficult, so, we assess the presence of EA using Aono's four point scale and the severity of EA using the PAED scale.

What causes the propofol to have no effect on preventing EA in our study? First, sevoflurane-related EA is affected by a combination of surgical procedures rather than the impact of propofol alone. The administration of propofol $1 \mathrm{mg} / \mathrm{kg}$ after sevoflurane anesthesia is effective on EA in Aouad's with strabismus surgery but this method is not effective in our study with adenotonsillectomy. Although the surgery type itself that involves the tonsil, middle ear, and eye are a major factor for inducing EA, adenotonsillectomy is the more important independent risk factor for EA than strabismus surgery [14]. Strabismus surgery may be related to visual disturbance. On the other hand, adenotonsillectomy may be associated with throat pain and bleeding irritation. Eckenhoff et al. [15] speculated that "sense of suffocation" during emergence from anesthesia may contribute to EA in patients undergoing head and neck surgery. Second, inadequate pain relief may be the stronger cause of EA than sevoflurane. Cohen et al. [16] reported a 2.5 $\mu \mathrm{g} / \mathrm{kg}$ dose of fentanyl minimized the incidence of EA to $18 \%$ after general anesthesia with sevoflurane. Bakhamees et al. [17] reported that the combination of low dose fentanyl before surgery and propofol at the end of surgery decreased the incidence and level of emergence agitation in children after adenotonsillectomy procedure under sevoflurane anesthesia. Kim et al. [18] reported that propofol $1 \mathrm{mg} / \mathrm{kg}$ at the strabismus surgery under sevoflurane anesthesia did not reduce EA in children, and they emphasized the importance of pain control. Ketorolac $1 \mathrm{mg} / \mathrm{kg}$ was administered to all children in both groups but it was not effective against EA, although it decreased the incidence EA in children undergoing myringotomy after sevoflurane anesthesia (38\% vs. 14\%) [19]. Compared with other studies, a smaller amount of analgesic agents were used in our study and additional analgesic drugs were not administered to children in the PACU. Nevertheless, CHIPP scales in this study were increasingly reduced than previous values as patient's recovery progressed. CHIPP scales were not influenced by propofol because propofol is not an analgesic agent. Third, there is a question about a larger dose than 1 $\mathrm{mg} / \mathrm{kg}$ propofol can be effective in preventing EA in children undergoing adenotonsillectomy. The duration of extubation was $13.7 \pm 3.8 \mathrm{~min}$ in the propofol group while the duration of extubation was $12.2 \pm 4.1 \mathrm{~min}$ in the saline group. The administration of propofol $1 \mathrm{mg} / \mathrm{kg}$ at end of surgery did not prolong the duration of extubation significantly compared with the saline group in our study. Although several studies reported that rapid awakening is an independent risk factor of EA [14, $20]$, delayed awakening with a large dose of propofol could increase adverse effects such as hypotension, bradycardia, apnea and close observation should be necessary. In addition, the effect of propofol in children undergoing painful procedures is still controversial and a study on the amount of propofol about incidence of EA has not been reported yet. Further investigations are necessary to assess the relation between the dose of propofol and the incidence of EA.

Many studies have shown that EA is self-limited and is resolved without pharmacologic intervention over time [12,14,20]. Our data also demonstrated that the incidence and severity of EA and pain intensity are improved over time without analgesic or sedative drugs. Our study allowed children to stay with one of parents in the PACU and this seemed to help them to acclimate themselves to a strange and naïve environment.

In our study, there was no significant decrease in the incidence and severity of emergence agitation after the administration of a single dose of $1 \mathrm{mg} / \mathrm{kg}$ propofol at the end of surgery in children undergoing adenotonsillectomy under sevoflurane anesthesia. However, the incidence and severity of emergence agitation significantly decreased over time and there was a statistically significant time effect. It is not recommended to administer 1 $\mathrm{mg} / \mathrm{kg}$ of propofol at the end of surgery to children who follow sevoflurane anesthesia for adenotonsillectomy in order to reduce emergence agitation. One of the limitations of this study was the difficulty in differentiating between pain and delirium. Considering the type of surgery, adequate analgesic modalities should be chosen to minimize postoperative pain. There is a need to further investigate the effects or benefits on different doses, different methods of administration, or additional analgesic treatments to examine the effects of propofol on emergence agitation in children receiving sevoflurane for adenotonsillectomy.

\section{Acknowledgements}

I sincerely thank my family and colleagues for their encouragement and support.

\section{References}

1. Lerman J. Sevoflurane in pediatric anesthesia. Anesth Analg 1995; 
81: S4-10.

2. Aono J, Ueda W, Mamiya K, Takimoto E, Manabe M. Greater incidence of delirium during recovery from sevoflurane anesthesia in preschool boys. Anesthesiology 1997; 87: 1298-300.

3. Kwak HJ, Kim JY, Kim JH, Kim YS, Park SY. The effect of ketamine and fentanyl on the incidence of emergence agitation after sevoflurane anesthesia in children undergoing tonsillectomy. Korean J Anesthesiol 2005; 49: 502-6.

4. De grood PM, Coenen LG, Van Egmond J, Booij LH, Crul JF. Propofol emulsion for induction and maintenance of anesthesia. A combined technique of general and regional aneshtsia. Acta Anaesthesiol Scand 1987; 31: 219-23.

5. Uezono S, Goto T, Terui K, Ichinose F, Ishguro Y, Nakata Y, et al. Emergence agitation after sevoflurane versus propofol in pediatric patients. Anesth Analg 2000; 91: 563-6.

6. Abu-Shahwan I. Effect of propofol on emergence behavior in children after sevoflurane general anesthesia. Paediatr Anaesth 2008; 18: 55-9.

7. Aouad MT, Yazbeck-Karam VG, Nasr VG, El-Khatib MF, Kanazi GE, Bleik JH. A single dose of propofol at the end of surgery for the prevention of emergence agitation in children undergoing strabismus surgery during sevoflurane anesthesia. Anesthesiology 2007; 107: 733-8.

8. Lapin SL, Auden SM, Goldsmith LJ, Reynolds AM. Effects of sevoflurane anesthesia on recovery in children: a comparison with halothane. Paediatr Anaesth 1999; 9: 299-304.

9. Cohen IT, Finkel JC, Hannallah RS, Hummer KA, Patel KM. Rapid emergence does not explain agitation following sevoflurane anaesthesia in infants and children: a comparison with propofol. Paediatr Anaesth 2003; 13: 63-7.

10. Nakayama S, Furukawa H, Yanai H. Propofol reduces the incidence of emergence agitation in preschool-aged children as well as in school-aged children: a comparison with sevoflurane. J Anesth 2007; $21: 19-23$.
11. Dahmani S, Stany I, Brasher C, Lejeune C, Bruneau B, Wood C, et al. Pharmacological prevention of sevoflurane- and desflurane-related emergence agitation in children: a meta-analysis of published studies. Br J Anaesth 2010; 104: 216-23.

12. Cole JW, Murray DJ, McAllister JD, Hirshberg GE. Emergence behaviour in children: defining the incidence of excitement and agitation following anaesthesia. Paediatr Anaesth 2002; 12: 442-7.

13. Sikich N, Lerman J. Development and psychometric evaluation of the pediatric anesthesia emergence delirium scale. Anesthesiology 2004; 100: 1138-45

14. Voepel-Lewis T, Malviya S, Tait AR. A prospective cohort study of emergence agitation in the pediatric postanesthesia care unit. Anesth Analg 2003; 96: 1625-30.

15. Eckenhoff JE, Kneale DH, Dripps RD. The incidence and etiology of postanesthetic excitment. A clinical survey. Anesthesiology 1961; 22: 667-73.

16. Cohen IT, Finkel JC, Hannallah RS, Hummer KA, Patel KM. The effect of fentanyl on the emergence characteristics after desflurane or sevoflurane anesthesia in children. Anesth Analg 2002; 94: 1178 81.

17. Bakhamees HS, Mercan A, El-Halafawy YM. Combination effect of low dose fentanyl and propofol on emergence agitation in children following sevoflurane anesthesia. Saudi Med J 2009; 30: 500-3.

18. Kim HJ, Kim HS, Kim SD, Kim CS, Kim JT, Lee KJ, et al. Effects of propofol and nalbuphine on emergence agitation after sevoflurane anesthesia in children for strabismus surgery. Korean J Anesthesiol 2008; 55: 575-8.

19. Davis PJ, Greenberg JA, Gendelman M, Fertal K. Recovery characteristics of sevoflurane and halothane in preschool-aged children undergoing bilateral myringotomy and pressure equalization tube insertion. Anesth Analg 1999; 88: 34-8.

20. Vlajkovic GP, Sindjelic RP. Emergence delirium in children: many questions, few answers. Anesth Analg 2007; 104: 84-91. 
Appendix 1. Aono's Four Point Scale

Calm

Not calm but could be easily consoled

Moderately agitated or restless and not easily calmed

Combative, excited, or disoriented, thrashing around

1

2

3

4

Appendix 2. Pediatric Anesthesia Emergence Delirium Scale

\begin{tabular}{ll}
\hline & \multicolumn{1}{c}{ Score } \\
\hline The child makes eye contact with the caregiver & $4:$ not at all \\
The child's actions are purposeful & $3:$ just a little \\
The child is aware of his/her surroundings & $2:$ quite a bit \\
& 1 : very much \\
& $0:$ extremely \\
\hline The child is restless & $0:$ not at all \\
The child is inconsolable & $1:$ just a little \\
& $2:$ quite a bit \\
& $3:$ very much \\
& $4:$ extremely \\
\hline
\end{tabular}

The scores of each item were summed to obtain a Pediatric Anesthesia Emergence Delirium (PAED) scale. The highest value of PAED scale is 20.

\section{Appendix 4. Ramsay Sedation Scale}

Patient is anxious and agitated or restless, or both

1

Patient is cooperative, oriented and tranquil

1

Patient responds to commands only

2

Patient exhibits brisk response to light glabellar tap or

loud auditory stimulus

Patients exhibits a sluggish response to light glabellar tap or

loud auditory stimulus

Patient exhibit no response

Appendix 3. Children and Infants Postoperative Pain Scale

\begin{tabular}{lll}
\hline \multicolumn{1}{c}{ Item } & \multicolumn{1}{c}{ Structure } & Points \\
\hline Crying & None & 0 \\
& Moaning & 1 \\
& Screaming & 2 \\
Facial expression & Relaxed/smiling & 0 \\
& Wry mouth & 1 \\
Posture of the trunk & Grimace (mouth and eyes) & 2 \\
& Neutral & 0 \\
Posture of the legs & Variable & 1 \\
& Rear up & 2 \\
& Neutral, released & 0 \\
Motor restlessness & Kicking about & 1 \\
& Tightened & 2 \\
& None & 0 \\
& Moderate & 1 \\
& Restless & 2
\end{tabular}

The scores of each item were summed to obtain a children and infants postoperative pain (CHIPP) scale. The highest value of CHIPP scale is 10 . 